\title{
The nature and origin of the Galactic center radio arc: a VLA Faraday study
}

\author{
Cornelia C. Lang ${ }^{1}$, James Toomey ${ }^{1}$, Dominic Ludovici ${ }^{1}$, Ann Mao $^{2}$ \\ and Mark Morris ${ }^{3}$ \\ ${ }^{1}$ Department of Physics \& Astronomy, University of Iowa, Iowa City, IA, United States \\ email: cornelia-lang@uiowa.edu \\ ${ }^{2}$ Department of Astronomy, University of Wisconsin, Madison, WI, United States \\ ${ }^{3}$ Department of Physics \& Astronomy, University of California, Los Angeles, CA, United States
}

\section{Motivation}

Despite their discovery almost 30 years ago, the origin of the Galactic center nonthermal filaments (NTFs) remains poorly understood. The improved capabilities of the VLA offer a fantastic opportunity to make a multi-frequency, full spectropolarimetric study of the radio arc at high angular resolution. Observations presented here are from DnC and $\mathrm{CnB}$ array configuration data taken at $\mathrm{S}, \mathrm{C}$ and $\mathrm{X}$ band (coverage between 2-12 $\mathrm{GHz}$; continuum only). In addition there are also Ka and Q band (continuum and spectral line coverage) observations that are part of the study but not shown in this short proceedings. These data will allow us to make the first high angular resolution Faraday study of the Galactic center radio arc.

\section{2. $\mathrm{S}$ band continuum image of the radio arc}

Figure 1 shows the first $\mathrm{S}$ band image of the radio arc at high angular resolution. Diffuse thermal structure can be seen throughout the continuum image as well as bright flux emanating from the NTFs across our observed frequency bands. Strikingly, several new point sources and a shell are detected. The spectral indices of the majority of the point sources indicate that they are background extragalactic sources.

\section{3. $\mathrm{C}$ and $\mathrm{X}$ band polarized intensity images of the radio arc}

Figure 2 shows that the radio arc is strongly polarized and that polarized intensity increases with frequency. The $\mathrm{X}$ band image (right) shows the most prominent polarized intensity. Additionally, there appears to polarization perpendicular to the NTFs. Current fractional polarization measurements are on the order of $26 \%$ at $\mathrm{C}$ band and more than $100 \%$ at $\mathrm{X}$ band. The large value for fractional polarization at $\mathrm{X}$ band is likely due to large scale flux missing in the total intensity.

\section{Future work: full Faraday study of the radio arc}

These are just the first, very preliminary results on a complex, multi-configuration data set. Future work includes: (1) determining the spectral index from 2-50 GHz, (2) imaging the polarized intensity to assess the fractional polarization and polarized intensity structure as a function of frequency, (3) determining the rotation measure toward the radio arc NTFs and carrying out a full Faraday synthesis study, and (4) making high 


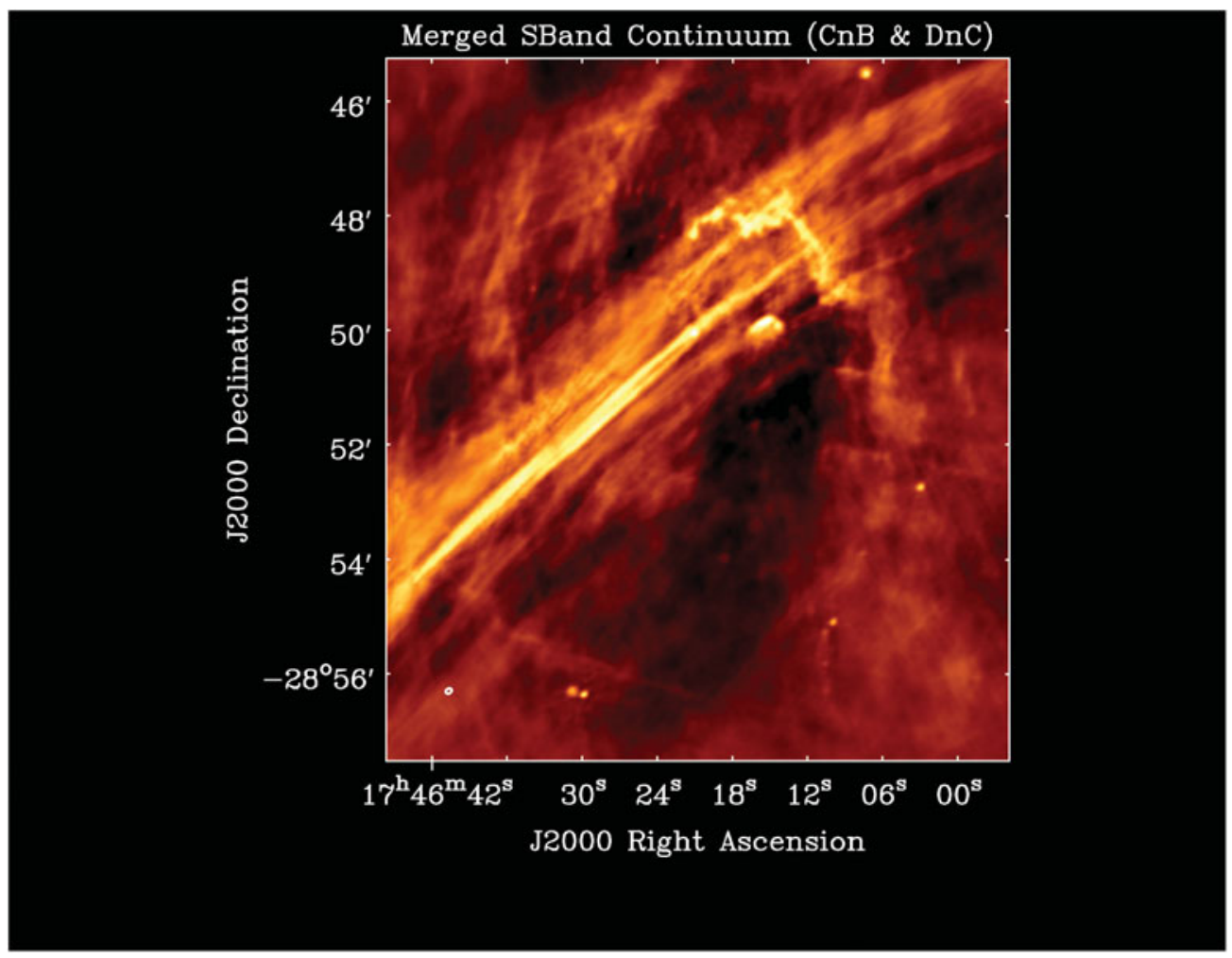

Figure 1. S band ( $\mathrm{CnB}$ and $\mathrm{DnC})$ continuum image between 2.5-3.5 GHz. This image has a spatial resolution of $6.70^{\prime \prime} \times 5.70^{\prime \prime}$ and an RMS noise of $29.88 \mu \mathrm{Jy} /$ beam. [A COLOR VERSion is AVAILAbLE ONLINE.]
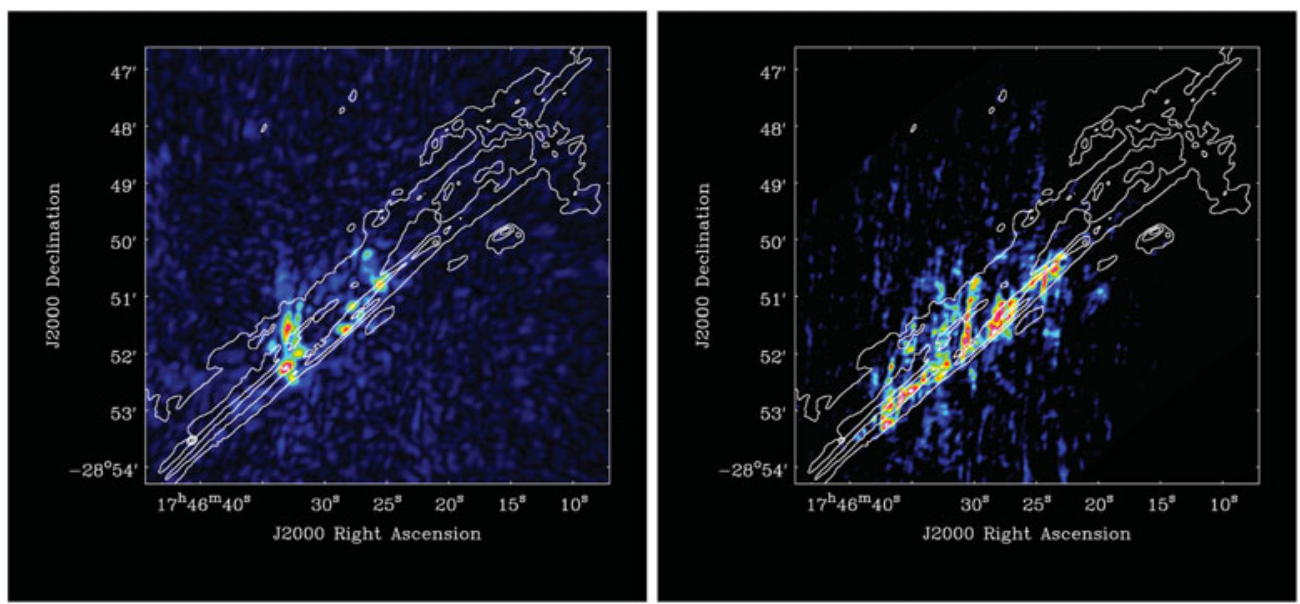

Figure 2. The radio arc exhibits polarization at low frequencies. Left: a C band (DnC) polarization map at 5.6-6.0 GHz with a spatial resolution of $11.29^{\prime \prime} \times 8.18^{\prime \prime}$ and an RMS noise of 0.2 $\mathrm{mJy} /$ beam. Right: an X band (DnC) polarization map at $10.8-11.1 \mathrm{GHz}$ with a spatial resolution of $6.83^{\prime \prime} \times 4.07^{\prime \prime}$ and an RMS noise of $0.2 \mathrm{mJy} / \mathrm{beam}$. [A COLOR Version is AVAllable online.] 
angular resolution images showing the intrinsic magnetic field orientation in the radio arc NTFs. The ultimate goal is to better understand the nature and origin of the radio emission in these enigmatic magnetized features that appear to be unique to the Galactic center. 